\title{
Acesso Vídeo-Laparoscópico no Tratamento Cirúrgico da Diverticulite Aguda
}

\section{The Vídeolaparoscopic Access in the Surgical Treatment of Acute Diverticular Disease}

\author{
AFONSO HENRIQUEDA SILVAE SOUSA JR.; ARCEU SCANAVINI NETO; \\ ANGELITAHABR-GAMA
}

SOUSA JR AHS, SCANAVINI NETO A, HABR-GAMAA. Acesso vídeo-laparoscópico no tratamento cirúrgico da doença diverticular dos cólons. Rev bras Coloproct, 2006;26(3): 341-347.

RESUMO: A revisão de conceitos baseada na literatura recente relacionada ao tratamento da doença diverticular dos cólons pelo acesso vídeolaparoscópico é apresentada ao lado das indicações de formas de tratamento clássicas. A dupla abordagem videolaparoscópica, imediata para tratamento da peritonite seguida da ressecção tornada eletiva é a modalidade nova na literatura, mas ainda não padronizada. Discutem os autores dados relativos a esta tática e de outros estudos que podem ampliar o emprego desta abordagem.

Descritores: Colectomia; colectomia laparoscópica; cirurgia minimamente invasiva; doença diverticular; diverticulite.

\section{INTRODUÇÃO}

A alta e crescente prevalência da doença diverticular dos cólons, que atinge cerca de $60 \%$ na população acima da sétima década de vida ${ }^{(1)}$, assim como os riscos inerentes aos episódios agudos de diverticulite aguda (DA), perpetuam a discussão quanto ao seu melhor manejo.

Por outro lado, a relativa baixa proporção de casos de diverticulite, torna interessante a revisão de literatura dirigida, por amplificar a análise de dados e rediscutir condutas clássicas.

O tratamento de quadro agudo da doença diverticular dos cólons, manifestado com infecção, está entre os tópicos que merecem revisão, em que pese constituir somente $1 \%$ ou menos de casuísticas de portadores de doença diverticular.

Da mesma forma, a discussão quanto ao diagnóstico, às medidas clínicas (jejum até recupera- ção do íleo paralítico, emprego de antibióticos, tipo de hidratação parenteral, repouso, prazos de observação cliínica até eventual mudança para a atitude cirúrgica) e até os meandros do raciocínio relativos à fisiopatologia são atuais, mas escapam ao escopo deste trabalho, estando disponíveis em revisões minuciosas e ilustrativas ${ }^{(2,3)}$.

\section{Indicação do tratamento cirúrgico}

Constitui o quadro agudo infeccioso da diverticulite aguda urgência médica que enseja variadas opções terapêuticas, uma vez que sua apresentação é também variada, em espectro contínuo desde a diverticulite propriamente dita - um divertículo e a região onde se encontra tornam-se substrato de infecção - até a peritonite fecal disseminada originada de comunicação da luz intestinal com a cavidade abdominal através de divertículo perfurado devido ao agravamento da infecção diverticular inicial. Assim, o quadro mais lo-

Disciplina de Coloproctologia do Departamento de Gastroenterologia do Hospital das Clínicas da Faculdade de Medicina da Universidade de São Paulo - Brasil. 
calizado da afecção pode ser tratado com medidas clínicas e se obter sucesso.

Apresentação mais grave, a de abscesso localizado identificado por método diagnóstico por imagem, merece consideração especial. Além de seu papel diagnóstico e na decisão terapêutica - ao indicar a necessidade de tratamento cirúrgico no caso de abscessos de mais de $5 \mathrm{~cm}$ de maior diâmetro - a tomografia computadorizada é ainda método terapêutico, ao guiar a drenagem percutânea de abscessos menores ou, ao menos, adiando a intervenção cirúrgica neste episódio de DA ${ }^{(3)}$. Função complementar deste método é diagnosticar, por sua maior sensibilidade, o pneumoperitônio que indica a necessidade do tratamento cirúrgico, como também estudar a via urinária, para o diagnóstico de fístula com a bexiga ou raro acometimento ureteral, indicando a necessidade de passagem de catéter ureteral intra-luminal para desobstruir a via urinária e manter a função do rim acometido.

Não se eliminando o foco infeccioso por medidas clínicas ou por punção esvaziadora, faz-se necessária intervenção cirúrgica de urgência.

Em princípio, a intervenção cirúrgica deve ser conduzida com o intuito de resolução do foco séptico, realizando-se a ressecção do segmento colônico acometido, incluindo o abscesso ou o flegmão. Saliente-se que os procedimentos de simples derivação devem ser realizados excepcionalmente, quando as condições clínicas não permitirem, mas sabendo-se que o processo infeccioso inflamatório ainda persistirá, talvez com menos gravidade. Neste momento do ato cirúrgico, deve-se tomar decisão quanto à reconstrução do trânsito: de imediato ou adiada para o segundo ato. Estudo recente mostrou que a idade ou a presença de peritonite purulenta difusa constituem fatores isolados que contra-indicaram ressecção, lavagem intestinal peri-operatória ou anastomose primária ${ }^{(4)}$. Neste estudo, as contra-indicações para anastomose primária foram peritonite fecal, falência de órgão, comprometimento imunológico (desnutrição, imunodeficiência primária ou adquirida, quimioterapia recente, uso de corticosteróides ou doença neoplásica extracolônica) e classificação de risco cirúrgico IV, segundo a American Society of Anesthesiologists. Nestes doentes, a melhor opção foi a operação de Hartmann, seguida da colectomia subtotal (e anastomose íleo-retal) ou simples derivação externa (colostomia).

A proposta por Hinchey e colaboradores em $1978^{(52)}$ estabelece sistematização da decisão cirúrgi- ca, baseada no grau de "extensão" da infecção. Assim, para o tratamento de casos de grau I ou II correspondentes ao acometimento infeccioso-inflamatório pericólico, com ou sem coleção de pus respectivamente, indica-se a ressecção e anastomose primária. Há tendência na literatura, diante de casos de graus III e IV, optar-se pela reconstrução do traânsito intestinal em segunda intervenção cirúrgica, finalizando-se a primeira à Hartmann (ressecção, derivação externa do remanescente proximal e sepultamento do reto).

Associado à diverticulite aguda pode ocorrer concomitantemente quadro obstrutivo, situação que foi estudada por Gooszen et al (2001) ao realizarem anastomose primária em 45 pacientes. No subgrupo de pacientes operados com obstrução intestinal associada (oito pacientes), observaram mortalidade relacionada à deiscência anastomótica em três pacientes, contrastando com o subgrupo complementar de 37 pacientes (sem quadro obstrutivo) em que somente um paciente apresentou deiscência que não evoluiu para óbito ${ }^{(51)}$. Pode-se concluir a partir destes dados que a obstrução intestinal constitui fator de risco para deiscência de anastomose e deve ser ponderada na decisão cirúrgica.

Do exposto depreende-se que o tratamento cirúrgico da diverticulite aguda sofre individualização, levando-se em consideração desde as características clínicas do paciente, da forma de manifestação da doença e da gravidade do quadro infeccioso instalado, além de outros concomitantes relativos a outras complicações da doença diverticular.

\section{Papel da videolaparoscopia}

Pela possibilidade de refinar o diagnóstico pela visão proporcionada pelo método videolaparoscópico, recentemente algumas casuísticas têm mostrado bons resultados com tratamentos menos ortodoxos do que a ressecção colônica na abordagem de urgência. Quase todas estas publicações listam menos de cinco pacientes assim tratados, oferecendo pouco material para discussão, menos ainda para apoiar mudança de diretriz.

Dentre elas, destaca-se, no entanto, a de Faranda et al (2000) na qual sugerem uma nova tática de operação em dois atos cirúrgicos. Na primeira, realiza-se laparoscopia diagnóstica, lavagem intensa da cavidade, aplicação de cola de fibrina sobre a área da perfuração ou processo inflamatório e drenagem da cavidade. Na segunda etapa, executa-se a colectomia 
videolaparoscópica, tornada então eletiva. Na casuística apresentada de 18 pacientes com peritonite, dos quais oito com grau de Hinchey III ou IV, quatro pacientes na primeira intervenção necessitaram de sutura (endoscópica), em seis realizou-se a cobertura com epíploon da área de perfuração e os demais, somente aplicação de cola biológica. A segunda etapa havia sido cumprida por 14 pacientes. A mortalidade foi nula, consideradas ambas intervenções, e a taxa de morbidade foi considerada satisfatória na segunda operação, sem deiscência em todos os pacientes ${ }^{(50)}$.

Outras casuísticas maiores - observados por outro prisma, no entanto - sugerem que a mortalidade tende a zero em pacientes mais jovens, ao passo que nos doentes acima de 50 anos há mortalidade de até $35 \%$ para os casos operados em caráter de urgência ${ }^{\text {(5) }}$. Como corolário pode-se justificar maior liberalidade para indicar o tratamento cirúrgico mais precoce (eletivo) para pacientes mais jovens, assim como o tratamento do quadro agudo de pacientes com mais de 50 anos pela estratégia de dupla abordagem videolaparoscópica. Cautela na adoção deste modo de decidir deve ser enfatizada, pois as casuísticas disponíveis ainda não permitem conclusão definitiva suficientemente apoiada em evidência científica de comprovação ampla.

Por outro lado, a experiência acumulada com o uso do acesso vídeo-laparoscópico no tratamento cirúrgico eletivo da doença diverticular constitui a melhor indicação para o uso desta via de acesso dentre as afecções coloproctológicas, segundo a opinião de diversos autores ${ }^{(7,8)}$. Não há dúvidas quanto à exeqüibilidade da ressecção de segmentos colônicos nesta afecção, aferida pela velocidade com que vem sendo reproduzida em diversos centros com experiência em cirurgia coloproctológica e vídeolaparoscópica.

Dados provenientes de séries mais numerosas ilustram os bons resultados desta indicação aplicada na forma eletiva, trazendo evidências que aqui servem ao propósito de, ao conhecê-los, lançar luz para o desafio próximo de delimitar seu emprego na cirurgia de urgência de diverticulite aguda.

\section{Fatores de exclusão}

Encontra-se, em algumas das casuísticas, a exclusão de pacientes com IMC $>35 \mathrm{~kg} / \mathrm{m}^{2}$ e antecedente de operação abdominal prévia (exceto colecistectomia, apendicectomia e histerectomia) ${ }^{(9)}$.

\section{Via de Acesso e Recidiva}

Estima-se que a recorrência após ressecção colônica em doença diverticular não complicada varie entre 3 e $13 \%{ }^{(40-46)}$.

A proporção de casos de recidiva após período de seguimento mínimo de cinco anos é estatisticamente semelhante após procedimentos vídeolaparoscópicos (3/79) ou por laparotomia (7/79) para doença diverticular ${ }^{(48)}$. Nesta mesma casuística, no entanto, algumas características do ato cirúrgico são significativamente distintas - apesar de não ter impacto sobre o resultado final: a flexura esplênica foi mobilizada mais vezes à laparotomia (41/79 vs 19/ 79 grupos pareados), o comprimento da peça foi maior à laparotomia $(18,3$ vs $16,1 \mathrm{~cm})$, havia processo inflamatório na margem proximal de ressecção de mais casos operados por vídeo-laparoscopia (21 $v s 4)$ e a anastomose foi realizada com o sigmóide distal - e não com o reto - em mais casos de laparotomia.

Estudo de 236 pacientes operados (59\% por acesso vídeo-laparoscópico) com seguimento médio de 67 meses (11 a 130 meses) analisou: tipo de acesso (laparoscópico ou aberto), porcentagem de casos com liberação do ângulo esplênico, tipo de anastomose realizada, comprimento da peça ressecada, presença de inflamação na margem proximal e nível da anastomose. Índice de recorrência detectado de 5\%, sendo o nível da anastomose o único fator estatisticamente significativo $(\mathrm{p}=0,03) .{ }^{(47)}$

Para a realização da colectomia, utilizaram em geral três trocártes de trabalho (dois à direita do paciente para uso do cirurgião e um à esquerda para o auxiliar) e mais um periumbilical para a câmera, com os pacientes em posição de Lloyd-Davies, apesar de ter sido observada preferência de um grupo por posicionar o paciente em decúbito lateral direito, facilitando a exposição da flexura esplênica, mas perdendose conseqüentemente exposição satisfatória na pelve. (10). Quanto à incisão para retirada da peça, duas opções foram mais citadas: incisão mediana infra-umbilical e incisão em quadrante inferior esquerdo ${ }^{\left({ }^{9}\right)}$, esta última não se realizando secção muscular para o acesso, levando a menor taxa de morbidade.

Encontram-se estudos comparando o procedimento totalmente laparoscópico, em que a anastomose é realizada sob visão laparoscópica ao vídeo assistido, em que a anastomose é realizada após exteriorização dos segmentos colônicos de modo idêntico à operação 
aberta. Não se demonstraram grandes vantagens da-

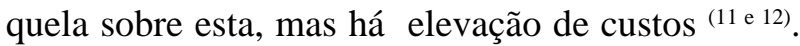

O desenvolvimento de dispositivos que permitem o acesso da mão do cirurgião à cavidade abdominal em procedimentos laparoscópicos tem merecido destaque na literatura nos últimos anos ${ }^{(13-16)}$. Tais materiais proporcionam a sensação tátil ao cirurgião e a manipulação direta das vísceras, mas atualmente encarecem em cerca de US\$ 700 o procedimento, embora aqueles que defendam sua utilização argumentem que o tempo operatório é menor e maior o conforto do cirurgião.

\section{Custos}

A análise dos custos envolvidos com procedimentos cirúrgicos constitui tarefa árdua e ingrata, devido à gama de fatores envolvidos. Um estudo que se preste a esta finalidade deve tentar abranger a maior variedade de custos diretamente envolvidos. Assim, custos de hospitalização, enfermagem, relativos aos equipamentos fixos da sala operatória, bem como aos descartáveis, ou consumíveis, custos com anestesia, laboratório, radiologia e farmácia e terapia intensiva ${ }^{(17)}$.

A colectomia laparoscópica tende a ser mais dispendiosa no que se refere à sala cirúrgica em função do custo do equipamento laparoscópico e do maior tempo utilizado, embora esta última variável não se confirme na maioria das séries em que, ao contrário, não se observou tempo cirúrgico muito aumentado em relação à operação aberta. Entretanto, este custo seria compensado com a abreviação do período de internação decorrente da redução no período pós-operatório.

Para ilustrar a divergência de dados entre os estudos de custo, citam-se alguns fatores que influenciariam na análise dos custos. Por exemplo, o tempo de internação tende a ser menor para as séries de doentes submetidos à laparoscopia ${ }^{(18-21)}$, mas em algumas séries este tempo é equiparável ${ }^{(22,23)}$. Este dado pode ser atribuído a menor tempo de íleo paralítico em algumas series ${ }^{(19,20,21,22,24,25,26)}$, mas que discordam de outras em que este tempo também é similar ${ }^{(23,27)}$. A taxa de complicações costuma ser apontada como semelhante ${ }^{(18,24,26)}$, se não menor que nas operações abertas ${ }^{(28)}$, com raras exceções ${ }^{(23)}$. Em relação à necessidade de medicação analgésica, a maioria dos estudos indicam ser menor na laparoscopia ${ }^{(24,25,26,28)}$, mas há series em que o consumo não foi significativamente diferente $^{(27)}$.
Um estudo atribuiu a redução de US\$ 450 por paciente em função de redução de custos com cuidados de enfermagem, farmácia e laboratório. Os autores ainda refletem quanto à diferença do impacto da redução do período de internação na dependência da rotatividade de leitos, ou seja, da diferença entre hospitais que trabalham com menor ou maior capacidade ociosa de leitos ${ }^{(17)}$. Naqueles, uma alta mais precoce libera leito para mais uma nova internação, onerando somente por custos fixos com pessoal. Já nos hospitais que trabalham com maior capacidade ociosa, a alta proporcionaria fechamento do leito, que poderia ocasionar fechamento de uma ala - levando a idéia ao extremo -, que reduziria os custos com pessoal.

Em estudos que analisaram exclusivamente doentes submetidos à colectomia por doença diverticular, há tendência a menores gastos nos grupos de laparoscopia ${ }^{(28,29)}$.

\section{Conversão}

Índices de conversão para operações colorretais variam em torno de $15 \%$, segundo dados de meta-análise envolvendo 3232 pacientes ${ }^{(39)}$. Para a DA realizada por acesso vídeo laparoscópico, de 6,8\% e $18 \%{ }^{(30)}$.

O evento da conversão é um momento delicado para o profissional envolvido diretamente com esta decisão. $\mathrm{O}$ aspecto relacionado à frustração da equipe cirúrgica envolve desde o transtorno causado à tática operatória, que em alguns casos faz surgir necessidade de reposicionamento do doente, nova anti-sepsia, recolocação de campos e troca de instrumental e se soma ao intuitivo aumento dos riscos e custos para o doente.

Nas primeiras séries que se prestaram à análise dos riscos inerentes à conversão, surgiram evidências de que a conversão resultasse em piores taxas de morbi/mortalidade para estes doentes, em comparação tanto com os doentes em que o procedimento laparoscópico havia sido concluído quanto com os que haviam se decidido previamente por acesso por laparotomia. Em 1658 pacientes operados por laparoscopia para tratamento de doença colorretal, houve aumento na morbi-mortalidade nos casos em que houve conversão: $47,7 \%$ e $3,5 \%$ versus $26,1 \%$ e $1,5 \%$ para casos sem conversão ${ }^{(31)}$. Este autor aponta o aumento no tempo operatório, a falha técnica que motivou a conversão e a gravidade da doença de base como responsáveis por estes aumentos. 
Outros autores enfatizam que a conversão precoce leva à redução destas taxas. Mesmo ocorrendo aumento significativo no tempo operatório nos doentes em que houve conversão e retardo na alta por mais três dias que os demais pacientes, não houve maior morbidade ${ }^{(32)}$. Contribuindo para esta interpretação, estudo da Cleveland Clinic propôs que o procedimento fosse dividido em etapas e que cada etapa fosse cumprida em tempo máximo estipulado. Caso contrário, o procedimento seria convertido. Assim, conseguiu-se que o tempo cirúrgico dos que completaram o procedimento pelo acesso vídeolaparoscópico não fosse tão diferente daqueles que foram submetidos à conversão. Conseqüentemente, observou-se que os pacientes em que houve conversão não tiveram tempo cirúrgico muito alargado ou evolução desfavorável em relação ao grupo de doentes em que se optou por colectomia aberta de princípio ${ }^{(9)}$, ao contrário de outras séries em que a conversão alterou negativamente a morbidade ${ }^{(31,33,34)}$. Desta forma, estimula-se o acesso laparoscópico em todos os casos de início, ressalvadas as contra-indicações, e propõe o uso deste sistema de separação dos procedimentos em etapas.

Algumas variáveis são importantes em relação aos fatores de risco para conversão, entre elas a experiência do cirurgião. Encontrou-se redução da taxa de conversão estudada entre o primeiro e o sétimo ano do emprego da vídeolaparoscopia em um Serviço: a taxa caiu de 44 para $2 \%{ }^{(32)}$. A curva de aprendizado é conceito associado aos procedimentos ditos avançados em videocirurgia; por ele, dominar-se-ia determinado número mínimo de procedimentos cirúrgicos após o qual se atingiria proficiência para reali- zação de tal procedimento com menores taxas de conversão ou complicação. Na cirurgia colo-retal alguns autores propõem um número entre 12 e 50 procedimentos ${ }^{(36,37,38)}$.

Outros trabalhos falharam em apontar fator preditivo para conversão entre as características dos pacientes, embora a obesidade seja aceita como um fator provável ${ }^{(31,34,35)}$.

Em relação às operações por doença diverticular, a taxa de conversão em meta-análise variou em decorrência da gravidade da doença. Quando a indicação cirúrgica foi para casos com peridiverticulite, estenose cólica, crises recorrentes, a taxa de conversão foi de 4,8\% e para os casos com abscesso, perfuração bloqueada, fístula ou sangramento de $18,2 \%{ }^{(39)}$.

\section{Considerações finais}

Neste panorama apresentado aqui para contribuir para a reflexão sobre dados disponíveis na literatura, houve a intenção de não ser completo, pois ainda o tratamento da diverticulite aguda não sofreu modificações importantes. O acúmulo de experiência e sua comunicação científica é que vão nortear modificações de condutas já consagradas. Do apresentado aqui, aliada à proporção de $33,9 \%$ de casos de doença diverticular operados - e coligidos no Registro Brasileiro da Sociedade Brasileira de ColoProctologia - dentre o total de casos operados por videolaparoscopia e acrescida dos casos ainda limitados - incluindo alguns dos autores - de abordagem na fase aguda por videolaparoscopia é que resultará a experiência necessária para amadurecer propostas de tratamento individualizado que envolvam a videolaparoscopia.

ABSTRACT: This review is based on standards of treatment for diverticular disease, both classical and new ones due to cumulated videolaparoscopic experience. The sequential double videolaparoscopic procedure for acute diverticulitis is discussed along with data from other studies supporting this novel modality of surgical treatment.

Key words: Colectomy; laparoscopic colectomy; minimally invasive surgery; diverticular disease, diverticulitis.

\section{REFERÊNCIAS}

1. Painter NS, Burkitt DP. Diverticular disease of the colon, a 20th century problem. Clin Gastroenterol 1975;4:3-21.

2. Schechter S, Nulvey J, Eisenstat TE. Management of uncomplicated acute diverticulitis. Dis Colon Rectum 1999; 42: 470-6.
3. Brengman ML, Otchy DP. Timing of computed tomography in acute diveerticulitis. Dis Colon Rectum 1998; 41: 102338.

4. Biondo S, Jaurrieta E, Jorba R, Moreno P, Farran L, Borobia F. Intraoperative colonic lavage and primary anastomosis in peritonitis and obstruction. Br J Surg 1997; 84: $222-5$ 
5. Biondo S, Parés D, Martí Ragué J, Kreisler E, Fraccalvieri D, Jaurrieta E. Acute colonic diverticulitis in patient under 50 years of age. Br J Surg 2002; 89: 1137-41.

6. Ouriel K, Schwartz SL. Diverticular disease in the young patients. Surg Gynecol Obstet 1983; 156: 1-5.

7. Berthou JC, Charbonneau P. Benefits of the laparoscopic approach are the improved early postoperation course and the reduction of parietal sequelae. Surg Endosc 1999; 13: 457-60.

8. Bruce CJ, Coller JA, Murray JJ. Laparoscopic resection for diverticular disease. Dis Colon Rectum 1996; 39(10 Suppl): S1-6.

9. Senagore AJ, Duepre HJ, Delaney CP, Brady KM, Fazio VW. Results of a standardized technique and postoperative care plan for laparoscopic sigmoid colectomy: a 30 month experience. Dis Colon Rectum 2003; 46: 503-9.

10. Ferzli SG, Sayad P, Cacchione RN. The lateral approach to laparoscopic sigmoid colon resection. J Am Coll Surg 2001; 193: 105-8.

11. Bernstein MA, Dawson JW, Reissman P, Weiss EG, Nogueras JJ, Wexner SD. Is complete laparoscopic colectomy superior to laparoscpic assisted colectomy? Am Surg 1996; 62: 507-11.

12. Darzi A, Super P, Guillou PJ, Morson JR. Laparoscopc sigmoid colectomy: total laparoscopic approach. Dis Colon Rectum 1994; 37: 268-71.

13. Mooney MJ, Elliott PL, Galapon DB, James LK, Lilac LJ, O'Reilly MJ. Hand-assisted laparoscopic sigmoidectomy for diverticulitis. Dis Colon Rectum 1998; 41: 630-5.

14. Bemelman WA, Ringers J, Meijer DW, De Wit WC, Bannenberg JJ. Laparoscopic-assisted colectomy with the dexterity pneumo sleeve. Dis Colon Rectum 1996; 39: S59-61.

15. Darzi A. Hand-assisted laparoscopic colorectal surgery. Surg Endosc 2000; 14: 999-1004.

16. Litwin DE, Darzi A, Jakimowicz J. Hand-assisted laparoscopic surgery (HALS) with the HandPort system: initial experience with 68 patients. Ann Surg 2000; 231: 715-23.

17. Delaney CP, Kiran RP, Senagore AJ, Brady K, Fazio VW. Case-Matched Comparison of Clinical and Financial Outcome After Laparoscopic or Open Colorectal Surgery. Ann Surg 2003; 238: 67-72.

18. Bemelman WA, Slors JF, Dunker MS. Laparoscopic-assisted vs open ileocolic resection for Crohn's disease. A comparative study. Surg Endosc 2000; 14: 721-5.

19. Young-Fadok TM, Radice E, Nelson H. Benefits of laparoscopic-assisted colectomy for colon polyps: a casematched series. Mayo Clin Proc 2000 Apr; 75: 344-8.

20. Kakisako K, Sato K, Adachi Y. Laparoscopic colectomy for Dukes A colon cancer. Surg Laparosc Endosc Percutan Tech 2000; 10: 66-70.

21. Franklin ME Jr, Rosenthal D, Abrego-Medina D. Prospective comparison of open vs. laparoscopic colon surgery for carcinoma. Five-year results. Dis Colon Rectum 1996; 39 (10 Suppl): S35-S46.
22. Khalili TM, Fleshner PR, Hiatt JR. Colorectal cancer: comparison of laparoscopic with open approaches. Dis Colon Rectum 1998; 41: 832-8.

23. Ho YH, Tan M, Eu KW. Laparoscopic-assisted compared with open total colectomy in treating slow transit constipation. Aust N Z J Surg 1997; 67: 562-5.

24. Joo JS, Amarnath L, Wexner SD. Is laparoscopic resection of colorectal polyps beneficial? Surg Endosc 1998; 12: 1341-4.

25. Young-Fadok TM, HallLong K, McConnell EJ. Advantages of laparoscopic resection for ileocolic Crohn's disease. Improved outcomes and reduced costs. Surg Endosc 2001; 15: 450-4.

26. Marubashi S, Yano H, Monden T. The usefulness, indications, and complications of laparoscopy-assisted colectomy in comparison with those of open colectomy for colorectal carcinoma. Surg Today 2000; 30: 491-6.

27. Bokey EL, Moore JW, Chapuis PH. Morbidity and mortality following laparoscopic-assisted right hemicolectomy for cancer. Dis Colon Rectum 1996; 39 (10 Suppl): S24-S8.

28. Kohler L, Rixen D, Troidl H. Laparoscopic colorectal resection for diverticulitis. Int J Colorectal Dis 1998; 13: 43-7.

29. Senagore AJ, Duepree HJ, Delaney CP. Cost structure of laparoscopic and open sigmoid colectomy for diverticular disease: similarities and differences. Dis Colon Rectum 2002; 45: 485-90.

30. Schwandner O, Farke S, Fischer F, Eckmann C, Schiedeck TH, Bruch HP.Laparoscopic colectomy for recurrent and complicated diverticulitis: a prospective study of 396 patients. Langenbecks Arch Surg 2004 Apr;389(2):97-103.

31. Marusch F, Gastinger I, Schneider C, Scheidbach H, Konradt J, Bruch HP. Importance of conversion for results obtained with laparoscopic colorectal surgery. Dis Colon Rectum 2001; 44: 207-14.

32. Le Moine, M-C1; Fabre, J-M2; Vacher, C2; Navarro, F1; Picot, M-C3; Domergue, J.Factors and consequences of conversion in laparoscopic sigmoidectomy for diverticular disease. Br J Surg 2003; 90: 232-6.

33. Slim K, Pezet D, Riff Y, Clark E, Chipponi J. High morbidity rate after converted laparoscopic colorectal surgery. Br J Surg 1995; 82: 1406-8.

34. Schwandner O, Schiedeck TH, Bruce $\mathrm{H}$. The role of conversion in laparoscopic colorectal surgery: do predictive factors exist? Surg Endosc 1999; 13: 151-6.

35. Schlachta CM, Mamazza J, Seshadri PA, Cadeddu MO, Poulin EC. Predicting conversion to open surgery in laparoscopic colorectal resections. A simple clinical model. Surg Endosc 2000; 14: 1114-7.

36. Agachan F, Joo JS, Sher M, Weiss EG, Nogueras JJ, Wexner SD. Laparoscopic colorectal surgery. Do we get faster? Surg Endosc 1997; 11: 331-5.

37. Senagore AJ, Luchtefeld MA, Mackeigan JM. What is the learning curve for laparoscopic colectomy? Am Surg 1995; 61: 681-5. 
38. Simons AJ, Anthone GJ, Ortega AE. Laparoscopic-assisted colectomy learning curve. Dis Colon Rectum 1995; 38 : 600-3.

39. Gervaz P, Pikarsky A, Utech M, Secic M, Efron J, Belin B. Converted laparoscopic colorectal surgery. Surg Endosc 2001; 15: 827-32.

40. Munson KD, Hensien MA, Jacob LN, Robinson AM, Liston WA. Diverticulitis: a comprehensive follow-up. Dis Colon Rectum 1996; 39: 318-22.

41. Wolff BG, Ready RL, MacCarty RL, Dozois RR, Beart RW Jr. Influence of sigmoid resection on progression of diverticular disease of the colon. Dis Colon Rectum 1984; 27: 645-7.

42. Bergamaschi R. Uncomplicated diverticulitis of the sigmoid: old challenges. Scand J Gastroenterol 1997; 32: 1187-9.

43. Benn PL, Wolff BG, Ilstrup DM. Level of anastomosis and recurrent colonic diverticulitis. Am J Surg 1986; 151: 269-71.

44. Bergamaschi R, Arnaud JP. Anastomosis level and specimen length in surgery for uncomplicated diverticulitis of the sigmoid. Surg Endosc 1998; 12: 1149-51.

45. Bergamaschi R, Arnaud JP. Intracorporeal colorectal anastomosis following laparoscopic left colon resection. Surg Endosc 1997; 11: 800-1.

46. Leigh JE, Judd ES, Waugh JM. Diverticulitis of the colon: recurrence after apparently adequate segmental resection. Am J Surg 1962; 103: 51-4.
47. Thaler K, Baig MK, Weiss EG, Nogueras JJ, Arnaud JP, Wexner $\mathrm{SD}$, et al. Determinants of recurrence after sigmoid resection for uncomplicated diverticulitis. Dis Colon Rectum 2003; 46: 385-8.

48. Thaler K, Weiss EG, Nogueras JJ, Arnaud JP, Wexner SD. Recurrence Rates at Minimum 5-Year Follow-up: Laparoscopic Versus Open Sigmoid Resection for Uncomplicated Diverticulitis. Surg Lap Endosc Perc Tech 2003;13(5):325-7.

49. Campos FG. Complications and Conversions in Laproscopic colorectal surgery. Results of a multicecnter brazilian trial. Surg Laparosc Endosc Per Techniques 2003; 13:173-9.

50. Faranda C, BArrat C, Catheline JM. Two-stage laparoscopic management of generalized peritonitis due to perforated sigmoid diverticula: eighteen cases. Surg Laparosc Endosc Percutan Tech 2000; 10: 135-8.

51. Gooszen AW, Tollenaar RA, Geelkerken RH. Prospective study of primary anastomosis following sigmoid resection for suspected acute complicated diverticular disease. Br J Surg 2001, 88:693-697.

52. Hinchey EJ, Schaal PG, Richards GK. Treatment of perforated diverticular disease of the colon. Adv Surg 1978, 12:85-109.

\section{Endereço para correspondência:} AFONSO HENRIQUE DA SILVA E SOUSA JR. Rua Coronel Cabrita, 13, apto 92 - Jd Glória

01545-030 - São Paulo - SP

E-mail: afonso_sousa@hotmail.com 